\title{
Haemorrhagic peritonitis as a late complication of echocardiography guided pericardiocentesis
}

\author{
H Luckraz, S Kitchlu, A Youhana
}

Heart 2004;90:e16 (http://www.heartjnl.com/cgi/content/full/90/3/el6). doi: 10.1136/hrt.2003.024075

Clinically significant pericardial effusion is an uncommon complication after cardiac surgery. Pericardiocentesis can be performed either through a mini-sternotomy or under echocardiography guidance. Echocardiography guidance is a relatively safe procedure and it avoids the need for another general anaesthetic. However, in this post cardiac surgical patient echocardiography guided pericardiocentesis was complicated several days later by haemorrhagic peritonitis.

$\mathrm{S}$ gnificant pericardial effusion occurs infrequently after cardiac surgery. ${ }^{1}$ Diagnosis by clinical findings alone is often a challenge. ${ }^{2}$ Echocardiography is considered the technique of choice for diagnosing pericardial effusion and echocardiography guided pericardiocentesis (EGP) is a relatively simple and safe treatment for this condition. ${ }^{2-4}$ Complications are rare but carry higher risk in patients who have undergone cardiac surgery than in patients with nonsurgical effusion. ${ }^{5}$ We describe the case of a patient who presented with a large pericardial effusion after cardiac surgery and who, after undergoing EGP, developed life threatening blood peritonitis several days after the procedure.

\section{CASE REPORT}

A 62 year old man who underwent urgent coronary artery bypass grafting and mechanical aortic valve replacement was discharged home after an uneventful postoperative recovery. He was given warfarin because of his mechanical valve (international normalisation ratio (INR) range 3.0-3.5). During his follow up outpatient visit four weeks postoperatively, he complained of tiredness, lethargy, and shortness of breath.

Haemodynamic examination was unremarkable except for an increased jugular venous pressure at $6 \mathrm{~cm}$. He was in sinus rhythm at 60 beats/min and was normotensive (130/ $80 \mathrm{~mm} \mathrm{Hg}$ with no pulsus paradoxus). Air entry was reduced in the right lower zone of the chest.

Haemoglobin concentration was $11.1 \mathrm{~g} / \mathrm{dl}$ and the INR was 3.7. ECG confirmed sinus rhythm. Chest radiography showed an enlarged cardiac shadow (fig 1) (cardiothoracic ratio 17.5:31.5). A transthoracic echocardiogram was performed, which showed significant pericardial effusion but no evidence of cardiac tamponade. The patient was admitted for pericardial drainage, which was carried out when the INR was 1.5. EGP through the subxiphoid approach drained $400 \mathrm{ml}$ of blood stained fluid. A pigtail catheter (5 French, Kimal Ref K 1760/2) was left in the pericardial space for continuous drainage. An additional $450 \mathrm{ml}$ of blood stained fluid was drained over the following 24 hours. The drain was removed and oral anticoagulation with warfarin was reinstituted. Intravenous heparin was also used while the INR was $<2$.

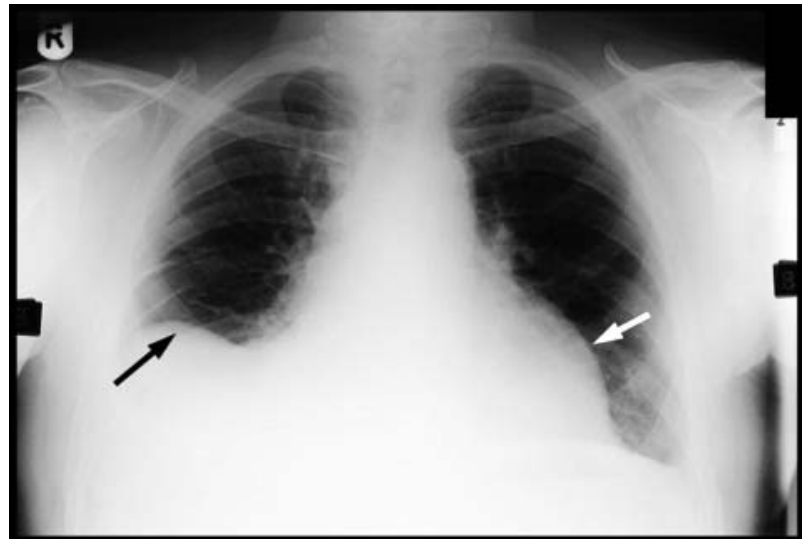

Figure 1 Chest radiograph before pericardiocentesis showing elevated right hemidiaphragm (black arrow) and enlarged cardiac shadow (white arrow).

On the third day after the procedure the patient complained of sickness and abdominal pain. On examination, he was found to be haemodynamically stable with mild tenderness over the epigastrium. His haemoglobin concentration was $10.7 \mathrm{~g} / \mathrm{dl}$. The INR was 1.4 and the activated partial thromboplastin time (APTT) ratio was 2.3. Over the following 12 hours, he developed generalised severe abdominal pain with guarding. He became haemodynamically unstable and his haemoglobin concentration dropped significantly $(7.4 \mathrm{~g} / \mathrm{dl})$. INR was 1.7 and APTT ratio was 1.8 . The patient was resuscitated with blood and fresh frozen plasma. Transthoracic echocardiogram did not show any significant pericardial collection. However, an abdominal ultrasound scan showed a considerable amount free fluid in the peritoneal cavity. Urgent exploratory laparotomy was performed. Four litres of blood was evacuated from the peritoneal cavity. There was no active bleeding point but puncture holes, surrounded by clots, were noted in the diaphragm. Haemostasis was secured and no other abnormality was detected at laparotomy. Thereafter, the patient made an uneventful recovery and was discharged home after stabilisation of his INR.

\section{DISCUSSION}

The use of EGP was first described in $1973^{6}$ and was popularised by Callahan and colleagues ${ }^{7}$ following their experience at the Mayo Clinic. Tsang and colleagues ${ }^{2}$ estimated that the incidence of significant postcardiotomy pericardial collection was about $0.8 \%$ with predisposing factors being previous valve surgery, use of anticoagulants, post-pericardiotomy syndrome, and autoimmune reactions. Data from the Mayo Clinic suggest that the success rate of EGP is over $95 \%$ but it does carry a $4.7 \%$ complication rate, ${ }^{1}$ which is much lower than with the blind approach. ${ }^{8}$ The 
major complications are damage to the cardiac chambers, laceration of intercostal vessels, pneumothorax, arrhythmias, infection, and death. Minor complications are transient entry in the cardiac chambers, vasovagal episodes, pleuropericardial fistula, and small pneumothorax. ${ }^{1}$ The classic subxiphoid approach for EGP has the lowest incidence of complications. ${ }^{3}$

It is very important to recognise that the postcardiac surgery pericardial fluid volume and pericardial pressure are not always linearly related. Thus, a patient may accumulate a large pericardial effusion with negligible haemodynamic sequelae. ${ }^{9}$ A large pericardial effusion has been, arbitrarily, defined as draining over $400 \mathrm{ml}$ during the initial aspiration. ${ }^{1}$

Echocardiography is generally accepted as the ideal diagnostic tool in the detection of pericardial effusion. ${ }^{4}$ EGP is a relatively simple and safe technique. ${ }^{2}$ As previously mentioned, one of the main factors predisposing to late pericardial fluid collection is the use of anticoagulant. In this setting, the pericardial fluid is often bloody and non-clotting, while it is straw coloured or serous in those who are not given an anticoagulant. ${ }^{10}$

In the present case pericardial effusion after cardiac surgery was clinically significant $(>400 \mathrm{ml})$. The patient had two of the predisposing factors-namely, valve surgery and administration of anticoagulants. He was treated by subxiphoid EGP, which drained $850 \mathrm{ml}$ of haemorrhagic fluid in 24 hours. Injury to the diaphragm during pericardiocentesis has been reported, ${ }^{5}$ but the development of haemoperitoneum requiring laparotomy has not been previously described in the literature. Moreover, the patient developed the life threatening complication on the third day after the procedure. This may have resulted from the additive effect of two anticoagulants (warfarin and heparin). As the patient was fully mobile, he may have dislodged some minor clots from his diaphragmatic injury sustained at the time of EGP. Fortunately, he was still an inpatient and his deteriorating condition was noted early and treated appropriately.

\section{Conclusion}

EGP remains the treatment of choice for drainage of pericardial effusion. However, for patients in the postcardiac surgery period and being given an anticoagulant, extra caution is needed to prevent major complications.

\section{Authors' affiliations \\ H Luckraz, S Kitchlu, A Youhana, Cardiothoracic Unit, Morriston \\ Hospital, Swansea NHS Trust, Morriston, Swansea, UK}

Correspondence to: Mr H Luckraz, Cardiothoracic Unit, Morriston Hospital, Swansea NHS Trust, Morriston, Swansea SA6 6NL, UK; HeymanLuckraz@aol.com

Accepted 2 October 2003

\section{REFERENCES}

1 Tsang TSM, Enriquez-Sarano M, Freeman WK, et al. Consecutive 1127 therapeutic echocardiographically guided pericardiocenteses: clinical profile, practice patterns and outcomes spanning 21 years. Mayo Clin Proc practice patterns and

2 Tsang TSM, Barnes ME, Hayes SN, et al. Clinical and echocardiographic characteristics of significant pericardial effusions following cardiothoracic surgery and outcomes of echo-guided pericardiocentesis for management. Chest 1999;116:322-31.

3 Vayre F, Lardoux H, Pezzano M, et al. Subxiphoid pericardiocentesis guided by contrast two-dimensional echocardiography in cardiac tamponade: experience of 110 consecutive patients. Eur J Echocardiogr 2000;1:66-71.

4 Fagan SM, Chan K. Pericardiocentesis blind no more. Chest 1999; 116:275-6.

5 Duvernoy O, Borowiec J, Helmius G, et al. Complications of percutaneous pericardiocentesis under fluoroscopic guidance. Acta Radiol 1992:33:309-13.

6 Goldberg BB, Pollack HM. Ultrasonically guided pericardiocentesis. Am J Cardiol 1973;31:490-3.

7 Callahan J, Seward J, Tajik AJ, et al. Pericardiocentesis assisted by twodimensional echocardiography. J Thorac Cardiovasc Surg 1983:85:877-9.

8 Krikorian JG, Hancock EW Pericardiocentesis. Am J Med 1978:65:808-14.

9 Harken AH, Hall AW, Hammond GL. The pericardium. In: Baue AE, Geha A, Hammond G, et al. Glenn's thoracic and cardiovascular surgery, 6th edn. Stamford: Appleton \& Lange, 1996:2299-310.

10 Russo AM, O'Connor WH, Waxman HL. Atypical presentation and echocardiographic findings in patients with cardiac tamponade occurring early and late after cardiac surgery. Chest 1993;104:71-8. 